\title{
Prevalence of psychosomatic and other medical illnesses in anorexic and bulimic patients ${ }^{1}$
}

\author{
A.-M. Ghadirian, F. Engelsmann, P. Leichner and M. Marshall \\ Department of Psychiatry, McGill University and Douglas Hospital Centre and Royal \\ Victoria Hospital, Montreal, Canada \\ Correspondence to: A.-M. Ghadirian, Douglas Hospital, 6875 LaSalle Blvd, Verdun \\ (Montreal), Quebec, Canada H4H 1R3
}

\begin{abstract}
The occurrence of psychosomatic disorders (PSD) in patients with anorexia nervosa and bulimia nervosa has rarely been investigated. We examined the prevalence of four psychosomatic disorders [peptic ulcer (PU), bronchial asthma (BA), neurodermatitis (ND), and rheumatoid arthritis (RA)] in patients consecutively admitted to an eating disorders (ED) program. Responses to a validated questionnaire and clinical file information were obtained from 152 patients, $60 \%$ of whom were bulimic and $40 \%$ were anorexic. The average age of the patients was 27.5 years and all but two patients were female. There was a higher life-time prevalence of the psychosomatic disorders among anorexics compared with bulimic patients, except for RA. PU and BA were reported by $10 \%$ of anorexics compared with $7.6 \%$ and $8.7 \%$, respectively, by bulimic patients. RA was rare in both anorexic $(1.6 \%)$ and bulimic patients $(5.4 \%)$. The young age of the patients under study was to be considered limiting the life-time prevalence. The study contains epidemiological data on the prevalence of other medical illnesses in ED patients as well as the prevalence of PSD and eating disorders in their families.
\end{abstract}

Keywords: Anorexia nervosa - Bulimia - Families - Medical illnesses - Prevalence - Psychosomatics

\section{INTRODUCTION}

Literature on the prevalence of psychosomatic disorders (PSD) in patients suffering from anorexia nervosa (AN) and bulimia nervosa (BN) is rare. Nor do we know about the pathogenic relationship between these two groups of disorders. Although AN and BN have been known for a long time, their phenomenology and prevalence have been particularly investigated in recent years.

Some researchers (Alexander, 1950) are of the opinion that anxiety related to repressed psychological conflicts plays an important role in the development of psychosomatic illness. Anxiety provokes autonomic responses which directly or indirectly cause alteration in physiological pattern or damage actual tissue. Others (Ross, 1954) have hypothesized that in psychotic patients such as schizophrenics, the capacity for repressing instinctual conflicts is diminished and therefore they are less likely to channel their distress into physiological symptoms. Psychosomatic disorders have been considered by some researchers to serve as a protection against psychosis (Schwartz and Semrad, 1951; Pedder, 1969). None of these theories, however, would explain why psychosomatic disorders can

\footnotetext{
' The paper was presented in the Scientific Program of the Canadian Psychiatric Association, October 1991.
}

occur in certain psychiatric patients more than in the others.

It could be assumed that eating disorder (ED) patients, particularly anorexics, may repress inner conflicts more intensely than psychotic patients. Therefore, it is possible that anorexic patients show greater prevalence of psychosomatic disorders. Some studies (Hall et al., 1989; Mitchell et al., 1991) point to the increased comorbidity of physical illness in ED patients. Mitchell et al. (1991) reported that bulimia nervosa was associated with a high rate of medical and psychiatric comorbidity. The reason for this elevated comorbidity remains unclear, and it is difficult to establish a cause-effect relationship.

Hall et al. (1989) in a prospective medical evaluation study of 276 hospitalized eating disordered patients reported a high rate (34\%) of medical disorders in bulimic patients, most of which were unknown to the patient or physician at the time of admission. These were moderately severe or serious medical disorders requiring treatment. When less serious illnesses were included, the total medical disorders rose to $70 \%$. Five per cent were considered severely ill. In patients with anorexia or anorexia alternating with bulimia, $10 \%$ suffered from severe life-threatening illness requiring intensive care unit (ICU) treatment. In 
view of an increasing prevalence of EDs and their clinical and psychosocial significance, there is a need to explore the psychosomatic disorders and other medical comorbidity in this population.

\section{Objectives}

This epidemiological study was intended to ascertain the prevalence of four psychosomatic illnesses; bronchial asthma (BA), peptic ulcer (PU), rheumatoid arthritis (RA), and neurodermatitis (ND), in a population of eating disorder patients. The prevalence of these psychosomatic disorders in the general population is as follows. Bronchial asthma may occur in $5 \%$ of the population of the USA, and over $50 \%$ of cases are diagnosed between the ages of 2 and 17 years (Daniele, 1988). Peptic ulcer affects 5-10\% of all individuals in their lifetime (Schiller, 1988). The prevalence of rheumatoid arthritis is roughly $1 \%$ in the general adult population. The peak incidence of its onset is between the 4th and 6th decade of life (Bennett, 1988). The precise prevalence of neurodermatitis, however, is not clear.

The study also evaluated the prevalence of other medical illnesses in ED patients. Moreover, the prevalence of PSD and ED in their families was also explored.

\section{METHODS}

\section{Sample}

The sample consisted of 152 patients who were consecutively admitted into the Eating Disorders Program of the Douglas Hospital Centre in 1989-1990. The sample represented the entire population of newly admitted patients (to out-patient clinic or in-patient unit) except those who (a) did not meet the DSM-III-R criteria for eating disorders or (b) declined to be interviewed for evaluation (four patients) after admission. The sample included in- and outpatients. The patients were self-referred or were referred by their treating physicians and other health professionals. Over $90 \%$ of the patients were evaluated by the same investigator (A.M.G.) using a validated questionnaire (Ramsay et al., 1982). The questionnaire contained variables measuring personal, demographic and clinical variables including the four selected psychosomatic disorders (PU, BA, ND and RA) and other systemic medical illnesses. These diagnoses were based on the clinical history of the patient, supported whenever possible by laboratory and/or radiological findings. Clinical evaluation included individual interview and information obtained from the patient's file.

Primary diagnosis. Of a total of 152 eating disorder (ED) patients, $60(40 \%)$ suffered from anorexia nervosa (AN) and $92(60 \%)$ from bulimia nervosa (BN). The sample was largely female with only two male anorexic patients.
Mixed clinical features. Fifteen per cent of the anorexic patients had bulimic episodes or features. Moreover $31.7 \%$ of the anorexic and $43.5 \%$ of the bulimic patients suffered from affective disorder (major depression, mostly non-psychotic), based on the DSM-III-R criteria (APA, 1987).

\section{Age}

The average age of the population under study $(n=152)$ was $27.5 \pm 7.0$ years. The age ranged from 17 to 53 years with a mode of 27 and a median of 26 years. The average age of the anorexic patients was slightly higher than the age of the bulimic patients: $28.2 \pm 7.1$ compared with $27.0 \pm 6.9$, respectively. There was no statistically significant age difference between these two diagnostic groups.

\section{Marital status}

The majority of the patients were single: $72 \%$ of the anorexics and $71 \%$ of the bulimics. Eighteen per cent of the anorexic patients were married compared with $9 \%$ of the bulimic patients. Seven per cent of the anorexic and $10 \%$ of the bulimic patients were separated or divorced.

\section{Education}

The population under study was well educated. The mean years of education for the two diagnostic groups was almost identical, 13.4 and 13.5, respectively, with a mode of 14 and a median of 13 years for the entire sample. Almost two-thirds of the patients had 12 or more years of education.

\section{Treatment status}

Four times more of the anorexic patients were hospitalized compared with the bulimic patients $(31.7 \%$ and $7.6 \%$, respectively). The discrepancy in the number of hospitalized patients was due to the severity of the physical and psychological conditions of the anorexic patients. Of the bulimic patients, 92 were out-patients compared with 67 of the anorexic subjects $\left[\chi^{2}(n=152)=19.2, p<0.001\right]$.

\section{Prior hospitalization}

Thirty-five per cent of the anorexics compared with $16.3 \%$ of the bulimics were previously hospitalized for eating disorders. Three anorexic and three bulimic patients (5\% vs $3.3 \%$ ) were previously hospitalized for psychosomatic disorders.

\section{Prior out-patient treatment}

Forty per cent of the anorexic and $34.8 \%$ of the bulimic patients had prior out-patient treatment for eating disorders. Thirteen anorexic and 17 bulimic patients $(21.6 \%$ vs $18.5 \%$ ) had prior out-patient treatment for other psychosomatic disorders. 


\begin{tabular}{|c|c|c|c|c|c|c|}
\hline & \multicolumn{3}{|c|}{ Anorexia $(n=60)$} & \multicolumn{3}{|c|}{ Bulimia $(n=92)$} \\
\hline & $n$ & $\%$ & Mean age & $n$ & $\%$ & Mean age \\
\hline Peptic ulcer (PU) & 6 & 10 & 24.8 & 7 & 7.6 & 21.7 \\
\hline Bronchial asthma (BA) & 6 & 10 & 10.8 & 8 & 8.7 & 16.8 \\
\hline Neurodermatitis (ND) & 7 & 11.7 & 17.7 & 8 & 8.7 & 8.1 \\
\hline Rheumatoid arthritis (RA) & 1 & 1.6 & 8.0 & 5 & 5.4 & 20.8 \\
\hline
\end{tabular}

TABLE II. Other current medical and psychiatric conditions

\begin{tabular}{|c|c|c|c|c|c|}
\hline & \multicolumn{2}{|c|}{ Anorexia $(n=60)$} & \multicolumn{2}{|c|}{ Bulimia $(n=92)$} & \multirow[t]{2}{*}{ Probability $(p)$} \\
\hline & $n$ & $\%$ & $n$ & $\%$ & \\
\hline None & 5 & 8.3 & 21 & 22.8 & 0.001 \\
\hline Cardiovascular & 9 & 15.0 & 5 & 5.4 & N.S. \\
\hline Gastrointestinal & 5 & 8.3 & 11 & 12.0 & 0.05 \\
\hline Respiratory & 5 & 8.3 & 1 & 1.1 & 0.02 \\
\hline Blood-lymphatic & 4 & 6.7 & 8 & 8.7 & N.S. \\
\hline Endocrine & 0 & & 2 & 2.2 & - \\
\hline Skin & 7 & 11.7 & 12 & 13.0 & N.S. \\
\hline Gynecological & 14 & 23.3 & 8 & 8.7 & 0.07 \\
\hline Musculoskeletal & 3 & 5.0 & 2 & 2.2 & N.S. \\
\hline Metabolic & 0 & & 1 & 1.1 & - \\
\hline Psychiatric depression & 5 & 8.3 & 18 & 19.6 & 0.001 \\
\hline Psychiatric other & 2 & 3 & 3 & 3.3 & N.S. \\
\hline
\end{tabular}

\section{Patients' present family}

A greater proportion of anorexic patients reported a loss of their father (deceased) $(n=14,23.3 \%)$ compared with the bulimic patients $(n=4,4.3 \%)$. Similarly, a greater proportion of the anorexic patients had lost their mother compared with the bulimic patients ( $11.7 \%$ vs $3.3 \%)$. Ten per cent of the anorexic and $6.5 \%$ of the bulimic patients reported a deceased brother while $5 \%$ of the patients in the two diagnostic groups indicated loss of a sister in the family. Three per cent of the bulimic patients had lost a child compared with none of the anorexic patients.

\section{Onset of eating disorder}

The mean age of the beginning of the eating disorder was $17.4 \pm 5.7$ years in the whole population under study. The disorder started earlier in life in the bulimic patients $(16.5 \pm 4.8$ years $)$ and later in the anorexic patients $(18.9 \pm 6.6$ years) $[t(150)=2.41, p<0.02]$.

\section{RESULTS}

\section{Psychosomatic disorders}

As Table I shows, there was a relatively higher lifetime prevalence of psychosomatic disorders in the anorexics than in the bulimics, with the exception of RA. However, this difference did not reach statistical significance. PU and BA were found in $10 \%$ of the anorexic patients com- pared with $7.6 \%$ and $8.71 \%$ in the bulimic patients, respectively. The RA prevalence was, however, lower in both the anorexic $(1.6 \%)$ and the bulimic patients $(5.4 \%)$. The young age of the patients under study has to be considered as a limitation of the life-time prevalence.

The onset of PU and ND was earlier in life for the bulimic patients (21.7 and 8.1 years) and later for the anorexic patients (24.8 and 17.7 years, respectively). The onset of BA and RA was earlier for the anorexic patients (10.8 and 8.0 years) and later for the bulimic patients ( 16.8 and 20.8, respectively). All the calculated $t$ values regarding the age of onset were not significant. The chi squares showed no statistical difference of the frequencies of the psychosomatic disorders in AN vs BN patients except for RA $\left[\chi^{2}(n=6)=4.9, p<0.02\right]$, using Yates' correction.

TABLE III. Family history of eating disorders

\begin{tabular}{lccccc}
\hline & \multicolumn{2}{c}{ Anorexic $(n=60)$} & & \multicolumn{2}{c}{ Bulimic $(n=92)$} \\
\cline { 6 - 7 } \cline { 5 - 6 } & $n$ & $\%$ & & $n$ & $\%$ \\
\hline Eating disorder in: & & & & & \\
$\quad$ Father & 2 & 3.3 & & 1 & 1.1 \\
Mother & 2 & 3.3 & & 3 & 3.3 \\
Brother(s) & 1 & 1.7 & & 1 & 1.1 \\
Sister(s) & 4 & 6.7 & & 11 & 12.0 \\
Children & 1 & 1.7 & & 0 & \\
\hline
\end{tabular}


TABLE IV. Family history of psychosomatic disorders

\begin{tabular}{|c|c|c|c|c|c|c|c|c|}
\hline & \multicolumn{2}{|c|}{ PU } & \multicolumn{2}{|c|}{ BA } & \multicolumn{2}{|c|}{ ND } & \multicolumn{2}{|c|}{ RA } \\
\hline & $n$ & $\%$ & $n$ & $\%$ & $n$ & $\%$ & $n$ & $\%$ \\
\hline \multicolumn{9}{|l|}{ Father } \\
\hline Anorexic pts & 12 & 20 & 2 & 3.3 & 3 & 5.0 & 0 & \\
\hline Bulimic pts & 7 & 7.6 & 6 & 6.5 & 1 & 1.1 & 10 & 10.9 \\
\hline \multicolumn{9}{|l|}{ Mother } \\
\hline Anorexic pts & 7 & 11.7 & 6 & 10 & 3 & 5 & 11 & 18.3 \\
\hline Bulimic pts & 3 & 3.3 & 6 & 6.5 & 1 & 1.1 & 12 & 13 \\
\hline \multicolumn{9}{|l|}{ Brother(s) } \\
\hline Anorexic pts & 2 & 3.3 & 5 & 8.3 & 2 & 3.3 & 0 & \\
\hline Bulimic pts & 2 & 2.2 & 11 & 12 & 2 & 2.2 & 1 & 1.1 \\
\hline \multicolumn{9}{|l|}{ Sister(s) } \\
\hline Anorexic pts & 2 & 3.3 & 5 & 8.3 & 0 & & 3 & 5 \\
\hline Bulimic pts & 2 & 2.2 & 5 & 5.4 & 2 & 2.2 & 0 & \\
\hline \multicolumn{9}{|l|}{ Children } \\
\hline Anorexic pts & 0 & & 2 & 3.3 & 2 & 3.3 & 0 & \\
\hline Bulimic pts & 0 & & 2 & 2.2 & 0 & & 1 & 1.1 \\
\hline
\end{tabular}

\section{General medical illnesses}

Table II shows that the bulimic patients had better health status than the anorexic patients, as $22.8 \%$ vs $8.3 \%$ reported no current condition. In general, ED patients seem to have many health problems. The anorexic women reported a high proportion of gynecological problems (23.3\%) compared with only $8.7 \%$ of the bulimic patients. The anorexic patients reported more cardiovascular and respiratory illnesses than the bulimic patients (15\% and $8.3 \%$ vs $5.4 \%$ and $1.1 \%$, respectively). The bulimic patients reported more gastrointestinal illnesses (12\%) and depression (19.6\%) compared with $8.3 \%$ and $8.3 \%$, respectively, for the anorexic patients.

\section{Prevalence of eating disorders and psychosomatic disorders in the family}

Anorexic patients reported a higher prevalence of eating disorders in their fathers compared with bulimics while bulimic patients revealed a greater prevalence of eating disorders among their sisters compared with anorexics. Both in anorexic and bulimic patients, 3.3\% of mothers suffered from eating disorders (Table III).

As Table IV shows, anorexic patients reported higher prevalence of PU in fathers, mothers and brothers compared with the bulimic patients: $20 \%, 11.7 \%$ and $3.3 \%$ vs $7.6 \%, 3.3 \%$ and $2.2 \%$, respectively. Bulimic patients reported higher prevalence of BA in fathers and brothers compared with the anorexic patients: $6.5 \%$ and $12 \%$ vs $3.3 \%$ and $8.3 \%$. The anorexic patients reported more prevalence of ND in fathers, mothers, brothers and children, but not in sisters. No anorexic patient reported RA in the father whereas $10.9 \%$ of the bulimic patients did. The anorexic patients reported RA in $18.3 \%$ of mothers compared with $13 \%$ of the bulimic patients' mothers.

\section{Certainty of diagnosis}

The evaluator rated the reliability of the data obtained on a scale from 1 (unreliable) to 5 (very strong evidence). The scale was validated and proven useful in previous international comparison of the prevalence of PSD in schizophrenic patients (Ramsay et al., 1982). The results of the current study showed very strong evidence and certainty of assessment indicated by the clinicians in $11 \%$ of the cases and strong evidence in $72 \%$ of the cases. An average rating score of 3.88 for anorexic patients and 3.83 for bulimic patients was high and satisfactory, showing no statistically significant difference of ratings between the two groups.

\section{DISCUSSION}

The results suggested that ED patients, particularly anorexics, showed an elevated prevalence of PSD (except for RA). These patients also showed a high prevalence of other medical and psychiatric comorbidities. Parents of anorexic patients had high prevalence of PU and this finding may have implications in the pathogenesis of eating disorders in the offspring.

Psychosomatic (psychophysiological) illnesses are believed to result, in general, from a number of interacting determinants: organic, psychological and environmental. Predisposition to PSD may be linked to chronic stress, genetic factors and inner conflicts. Although the psychological theories of PSD remain tentative and speculative (Ramsay et al., 1982), our approach is based on the belief that psychosocial factors may play a role in the onset and the course of certain illnesses, some of which are traditionally referred to as "classic PSD". The approach is concerned with reactions to and coping with life stresses which are disturbing for some but not for others at specific 
points in life and within a specific context. Psychologically, inner conflicts and struggle to control body image are important contributing factors in eating disorders. Social and cultural pressure for women to stay thin and follow the social feminine models create distress which could develop into psychological symptoms. The stressful impact of nutritional restriction or starvation and the distressing binge/purge cycles may precipitate biological and psychological effects on these patients.

Our findings on the prevalence of PSD in AN and BN patients can be of special significance in view of the scarcity of literature in this field. We feel that more vigorous research studies are warranted to assess the prevalence and mechanism of development of PDS in the eating disorder population.

\section{REFERENCES}

Alexander F (1950) Psychosomatic Medicine. Norton, New York.

American Psychiatric Association (1987) Diagnostic and Statistical Manual of Mental Disorders (DSM-III-R). APA, Washington, DC.

Bennett JC (1988) Rheumatoid arthritis. In: Cecil Textbook of
Medicine (Eds JB Wyngaarden and LH Smith), pp. 19982004. W.B. Saunders, Philadelphia.

Daniele RP (1988) Asthma. In: Cecil Textbook of Medicine (Eds JB Wyngaarden and LH Smith Jr), p. 403. W.B. Saunders, Philadelphia.

Hall RCW, Hoffman RS, Beresford TP et al. (1989) Physical illness encountered in patients with eating disorders. Psychosomatics, 30(2), 174-191.

Mitchell JE, Specker SM and De Zwaan M (1991) Comorbidity and medical complications of bulimia nervosa. Journal of Clinical Psychiatry, 52(10) (suppl.), 13-20.

Pedder JR (1969) Psychosomatic diseases and psychoses. Journal of Psychosomatic Research, 13, 339.

Ramsay RA, Ananth J, Engelsmann F, et al. (1982) Schizophrenia and psychosomatic illness. Journal of Psychosomatic Research, 26, 33-42.

Ross WD (1954) Psychosomatic disorders and psychoses. In: Recent Developments in Psychosomatic Medicine (Eds ED Wittkower and RA Cleghorn), pp. 397-418. Pitman, London.

Schiller LR (1988) Epidemiology, clinical manifestations and diagnosis (peptic ulcer). In: Cecil Textbook of Medicine (Eds JB Wyngaarden and LH Smith), p. 696. W.B. Saunders, Philadelphia.

Schwartz J and Semrad EV (1951) Psychosomatic disorders in psychoses. Psychosomatic Medicine, 13, 314.

(Received 21 May 1993; accepted 18 June 1993) 


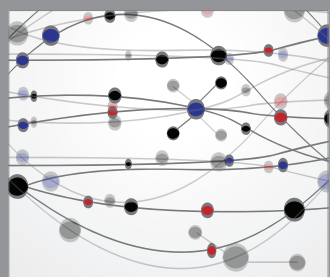

The Scientific World Journal
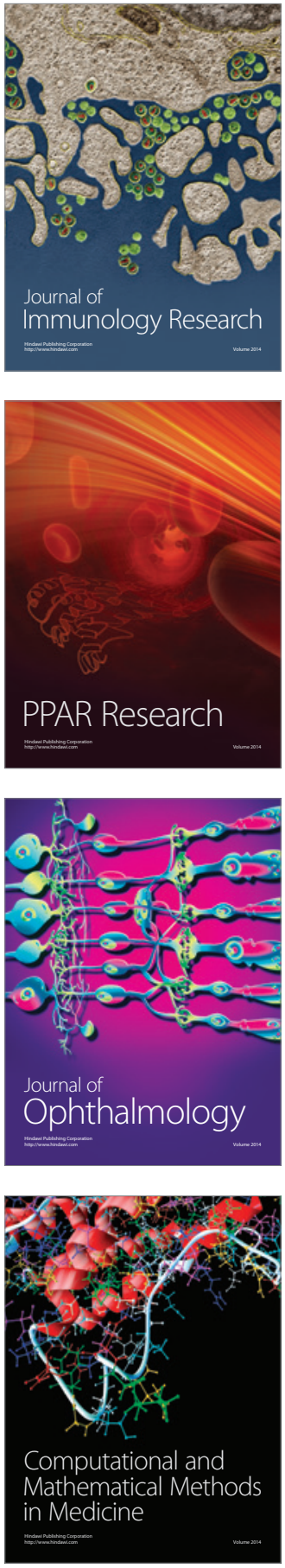

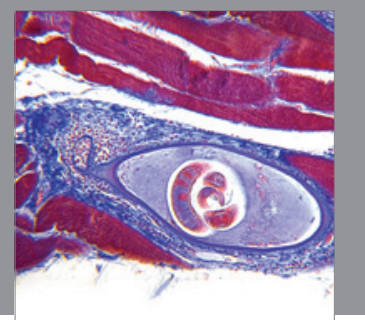

Gastroenterology

Research and Practice
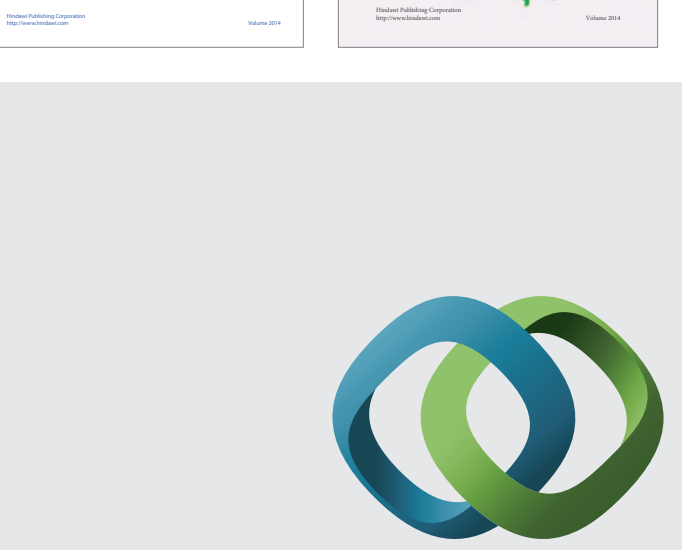

\section{Hindawi}

Submit your manuscripts at

http://www.hindawi.com
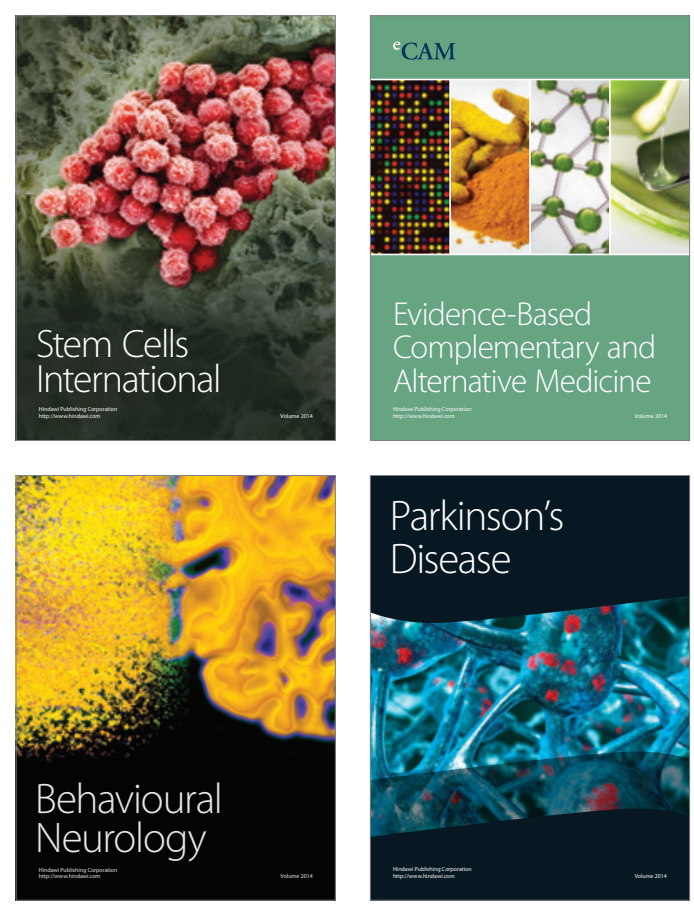

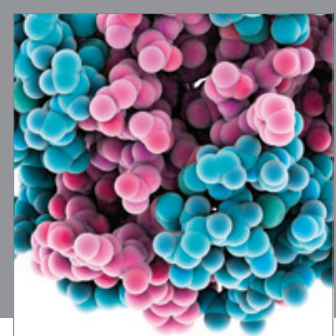

Journal of
Diabetes Research

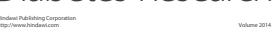

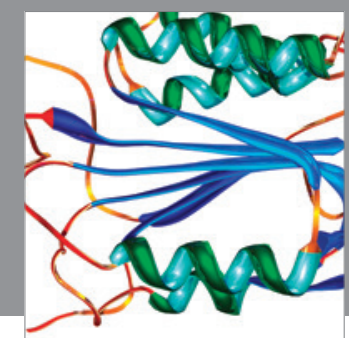

Disease Markers
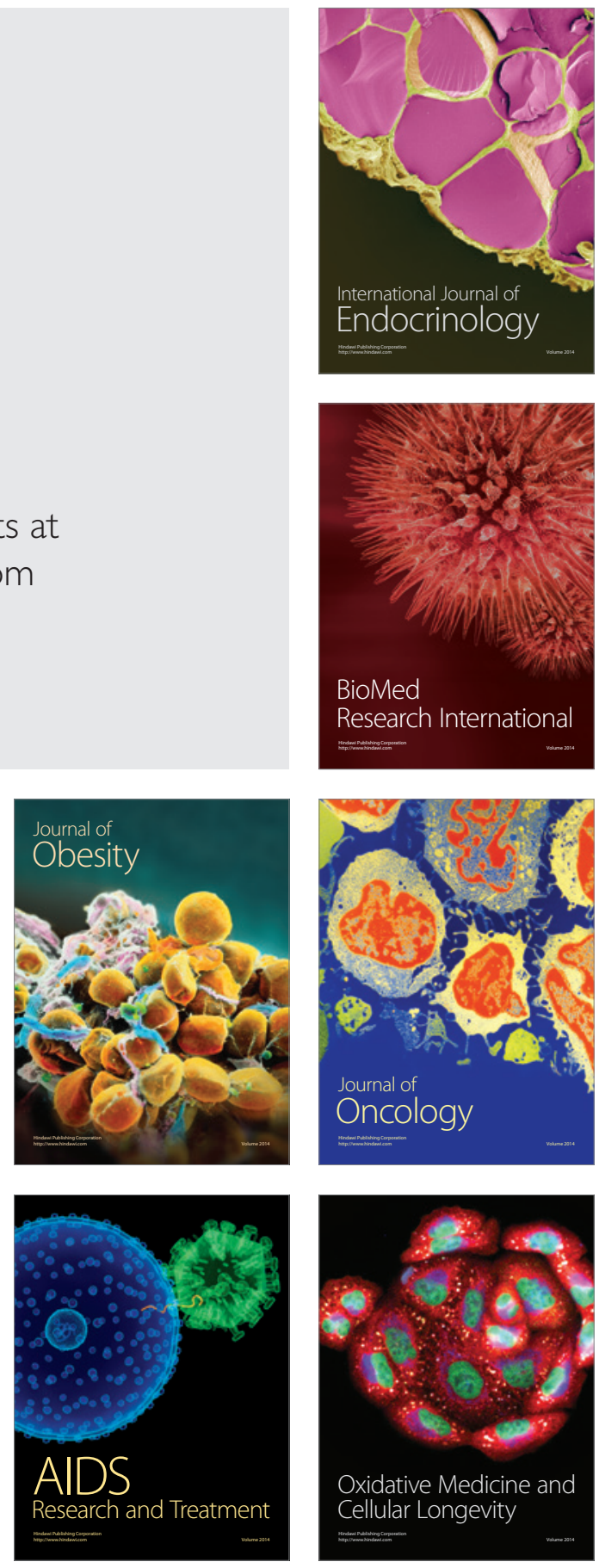